\title{
CERTIFICATION ET TRACABILITE Certification des semences de plantes oléagineuses
}

\section{Certification and traceability The certification of oil seed crops: the French example}

Oléagineux, Corps Gras, Lipides. Volume 8, Numéro 5, 510-3, Septembre - Octobre 2001, Dossier : Aspects des filières semencières Nord/Sud

Auteur(s) : Vincent POUPARD, Groupement national interprofessionnel des semences (GNIS), 44, rue du Louvre, 75001 Paris, France.

Résumé : Dès les années 50 , les organisations professionnelles avaient pris des initiatives pour garantir la qualité des semences commercialisées en France. Dans les années 60, ces initiatives ont été relayées par la réglementation publique nationale. En 1962, la certification devenue obligatoire a été confiée par le ministère de l'Agriculture au Groupement national interprofessionnel des semences et plants (GNIS). Au sein du GNIS, cette mission est assurée par le Service officiel de contrôle et de certification (SOC). Le GNIS, lieu de concertation entre les professionnels de la filière semences et les Pouvoirs publics, favorise l'évolution du système de certification. Les Pouvoirs publics voulaient ainsi garantir la qualité des semences pour les agriculteurs utilisateurs et favoriser le progrès génétique. Les caractéristiques de ce système ont évolué depuis 1966, au fur et à mesure de l'entrée en vigueur des directives communautaires de commercialisation pour chacune des espèces de grandes cultures, dont l'objectif était d'harmoniser les règles de certification et de commercialisation afin de permettre une libre circulation des semences dans l'Union européenne.

Summary : In the sixties, the certification of seeds was made compulsory in Europe for marketing purposes, to ensure consistent seed quality for farmers and allow free movement within the European Union. The GNIS (Groupement national interprofessionnel des semences et plants) was entrusted with official certification, which is carried out by its technical service, the SOC (Service officiel de contrôle et de certification). The French certification scheme gradually evolved to take into account, under official control, some of the means for quality management set up by the seed companies. Certification today guarantees the varietal conformity of seeds, as well as some technological and sanitary characteristics. It still uses very little the new laboratory methods developed by biotechnologies, but the SOC is studying their potential use to simplify present procedures, to accelerate decisions, and even to integrate guarantees on additional characteristics to meet the increasing expectations of consumers concerning quality and traceability.

Keywords : seeds, certification, GNIS, SOC. 


\section{ARTICLE}

\section{Certification des semences}

La certification des semences est une garantie officielle de conformité de produit qui comprend trois aspects : une certification variétale, une certification technologique et, dans certains cas, une certification sanitaire. Elle atteste, par ailleurs, que les semences ont suivi un processus de production normalisé et subi des contrôles de qualité prédéfinis. La réalité de ces contrôles est matérialisée par un certificat officiel apposé sur chaque emballage de semences.

* La certification variétale couvre l'identité et la pureté variétales des semences. Elle découle essentiellement du contrôle de la filiation et de la vérification des facteurs de production qui doivent exister au niveau de la parcelle. C'est le contrôle des cultures ou l'inspection sur pied.

* La certification technologique garantit la pureté spécifique et la faculté germinative ainsi que la teneur maximale en eau pour certaines espèces. Elle résulte de vérifications réalisées en laboratoire, selon des protocoles d'analyse normalisés.

* La certification sanitaire relève à la fois de l'inspection des cultures et de vérifications en laboratoire.

La certification est conduite sur la base des règlements techniques. Ces règlements s'appuient sur les obligations minimales des directives européennes et les exigences techniques et administratives complémentaires établies en accord avec les différentes familles professionnelles, approuvées par le ministère de l'Agriculture.

Progressivement, le système français de certification officiel a intégré ou s'est inspiré des pratiques d'autres secteurs d'activité, notamment de l'industrie, qui réalisaient également des contrôles de la qualité ou étaient soumis à une certification. Cette adaptation permanente a toujours cherché à :

- concilier les procédures techniques et les contraintes liées au caractère industriel et commercial de l'activité semences des entreprises;

- responsabiliser les entreprises et les organismes professionnels dans les procédures de contrôle de la qualité ;

- optimiser les coûts pour l'ensemble de la filière semences et les utilisateurs. Cette optimisation des moyens et des coûts a conduit le SOC à agréer, pour des opérations de contrôles officiels, des agents extérieurs au service, qu'ils soient salariés d'entreprises semencières ou d'organismes professionnels impliqués dans la production de semences. C'est le cas pour les principales opérations concernant le contrôle des cultures, l'échantillonnage des lots, l'analyse des échantillons et l'étiquetage officiel des emballages. Ces opérations sont validées en culture par sondage direct du SOC dans les parcelles de multiplication, et en usine sur les lots de semences.

\section{L'inspection des cultures : pour garantir la conformité variétale des semences}

Pour le secteur des oléagineux, les notations des productions de semences de prébase et de base sont assurées par des techniciens d'entreprises semencières agréés par le SOC. L'ensemble de ces productions fait l'objet de contrôles par des agents du SOC. Dans le cas de la dernière génération « 
semences certifiées ", les notations sont déléguées par le GNIS à I'ANAMSO (Association nationale des agriculteurs multiplicateurs de semences oléagineuses) et sont réalisées par des techniciens d'entreprises ou des agents de I'ANAMSO. Le SOC contrôle alors par sondage, pour chaque technicien, environ $20 \%$ des productions dont il a en charge le suivi (figure).

L'agrément des techniciens par le SOC intervient à l'issue d'une formation spécifique et d'une qualification.

Chaque parcelle de production fait l'objet d'un suivi par un technicien pendant la période de végétation, avec un nombre minimum de visites obligatoires à réaliser fixé par le règlement technique de l'espèce concernée. Ainsi, pour le tournesol, trois visites minimum sont obligatoires, une avant floraison, une courant floraison et une à maturité. L'objet de ces visites est de s'assurer que les parcelles satisfont au cahier des charges fixé par le règlement technique. Les contrôles réalisés concernent essentiellement :

- les distances d'isolement entre la parcelle de production et les parcelles avoisinantes de la même espèce ou d'espèces susceptibles de polliniser la culture porte-graine ;

- la vérification de l'identité du matériel produit, ou des lignées parentales dans le cas d'un hybride, par référence à la description morphologique réalisée sur ce matériel au moment de son inscription au catalogue (description réalisée en France par le Geves, Groupe d'étude et de contrôle des variétés et des semences) ;

- la vérification des numéros de lots de semences mères utilisés par l'agriculteur multiplicateur pour l'implantation de sa production de semences (contrôle de la filiation) ;

- la vérification de la pureté variétale du matériel produit. Toute plante différente de la variété produite est considérée comme hors type et n'est tolérée que dans des proportions très faibles ou doit donner lieu à des épurations avant floraison ;

- pour certaines espèces, la vérification du précédent cultural. Par exemple, les parcelles de production de semences de colza ne doivent pas avoir été emblavées avec des productions de crucifères pendant les sept années précédentes, afin de limiter les risques de repousses ;

- pour les espèces hybrides, la validation de la bonne concordance de floraison entre le parent mâle et le parent femelle ;

- la vérification de l'absence d'adventices pouvant poser des problèmes de pollution des lots et difficiles à éliminer ultérieurement lors du process de fabrication à l'usine ;

- l'état sanitaire de la parcelle. Ainsi, en tournesol, les plantes attaquées par le mildiou et le sclérotinia sont systématiquement retirées du champ et détruites.

Le technicien agréé est chargé de reporter l'ensemble de ses observations à l'issue de chacune de ses visites sur un document du SOC. Ce document, à caractère officiel, constitue une véritable carte d'identité reprenant tout l'historique de la parcelle de production de semences. 
En cas de non-conformité de la parcelle sur certains facteurs de production, le technicien peut être amené à demander à l'agriculteur multiplicateur de réaliser des travaux sur la parcelle (épuration, broyage, traitement, etc.).

À l'issue des visites de notation, le technicien transmet au SOC une proposition de classement des parcelles. Le SOC valide ces propositions et décide alors du devenir des récoltes (acceptation ou refus éventuel de tout ou partie de la production).

Les contrôleurs du SOC réalisent par sondage des visites destinées à s'assurer du bon suivi des parcelles par les techniciens agréés, de la réalisation des éventuels travaux demandés et de la bonne tenue des documents de notation.

\section{Le contrôle variétal}

Le contrôle variétal consiste à valider l'identité et la pureté variétales des lots produits. Cette validation se fait sur des échantillons représentatifs des lots, semés en microparcelle, par référence au standard de la variété, qui correspond au matériel référencé à l'issue de son inscription, détenu par l'agence du pays d'inscription de la variété.

Ces contrôles variétaux sont réalisés sur $100 \%$ des semences de base et par sondage sur la génération "semences certifiées ". Dans le cas des semences de base, cela correspond également à un contrôle simultané des lots utilisés dans les productions de semences certifiées mises en terre. Cette opération vise alors, d'une part, à valider l'identité du matériel par rapprochement avec le standard et, d'autre part, à obtenir une information précoce sur la pureté variétale afin d'informer, en cas de problème, les entreprises concernées, les techniciens chargés des notations et d'orienter les contrôles du SOC sur les productions concernées.

Dans le cas de semences certifiées, le contrôle variétal permet de mesurer la qualité des semences mises sur le marché mais également de donner des indications sur la qualité et la fiabilité du système de délégation des notations ainsi que sur l'adéquation des règles de production.

\section{La fabrication des lots en usine : assurer la traçabilité et les caractéristiques technologiques des semences}

À l'issue des récoltes, chaque lot d'agriculteur individualisé est livré dans une usine de production de semences agréée par le SOC. La semence va alors suivre un process de fabrication (séchage, nettoyage, calibrage, traitement, etc.). Dans certains cas, les différentes productions vont être associées en fonction de critères propres à l'entreprise et de façon à obtenir une homogénéité du produit et des caractéristiques du lot (calibre, germination, poids de mille grains, etc.). À chaque étape de ce process, la semence est tracée de façon à ce qu'on puisse, en bout de chaîne de fabrication, remonter à l'origine de la parcelle ou du groupe de parcelles ayant servi à constituer le lot final. Ce lot est ensuite échantillonné et analysé en laboratoire pour vérifier la conformité de ses caractéristiques par rapport aux normes de certification (germination, humidité, teneur en autres espèces, état sanitaire). 
Là encore, les opérations d'échantillonnage du lot de semences et d'analyse ont été progressivement déléguées aux opérateurs professionnels. Cette délégation se fait sous réserve d'une qualification préalable des agents chargés de l'échantillonnage et des analyses, et d'un agrément du matériel d'échantillonnage et du laboratoire, afin de s'assurer de la présence du matériel et du personnel nécessaires à la réalisation des analyses.

Par ailleurs, pendant toute la période d'activité de conditionnement des semences, le SOC réalise par sondage des prélèvements et des analyses officiels destinés à s'assurer en permanence que l'échantillonnage est correctement réalisé et que le laboratoire habilité de l'entreprise travaille de la même manière que le laboratoire officiel (SNES, Station nationale d'essais de semences). L'ensemble de ces contrôles fait chaque année l'objet d'une synthèse dans une commission du ministère de l'Agriculture qui décide ou non de la reconduction des opérations ainsi déléguées aux entreprises.

Cette conception française du système de certification des semences s'appuyant, pour une grande majorité de ses étapes, sur des opérations déléguées, sous contrôle officiel, aux opérateurs professionnels a fait ses preuves. Le pourcentage de refus en culture et sur lot de semences ne représente que 2 à $3 \%$ de la production. II a ainsi permis une adaptation aux contraintes techniques et économiques des entreprises tout en préservant sa mission essentielle de garantir la qualité vis-àvis des utilisateurs.

\section{Intérêt des nouvelles méthodes de laboratoire pour la certification}

L'évaluation de l'identité et de la pureté repose essentiellement aujourd'hui sur l'observation visuelle des caractères morphologiques des plantes, pour les caractères décrits par I'Upov (Union internationale pour la protection des obtentions végétales) sur lesquels s'appuient les études d'inscription des variétés.

L'augmentation du nombre des variétés inscrites et protégées a rendu de plus en plus difficile la possibilité de distinguer les variétés entre elles ou de les identifier précisément en parcelles. Les discussions actuelles en cours au niveau de l'Upov visent à étudier la possibilité de prendre en compte de nouveaux caractères, en particulier les caractéristiques biochimiques ou moléculaires (électrophorèse, sondes moléculaires). Le SOC sera donc amené à prendre en compte ces nouvelles caractéristiques, dès lors qu'elles feront partie intégrante de la description des variétés.

L'électrophorèse est déjà utilisée dans certaines situations, afin de clarifier des situations douteuses à l'issue des contrôles en culture, pour l'évaluation de l'identité ou de la pureté variétale. Des études sont également en cours pour essayer de mesurer l'intérêt des techniques de marquage moléculaire dans les procédures de certification.

Cependant, les évolutions en cours doivent pouvoir être applicables dans le cadre de la certification, sans générer de coûts ou de délais supplémentaires incompatibles avec l'économie du marché des semences.

Le système de certification actuel repose essentiellement, pour l'identité et la pureté variétales, sur une vérification des moyens de production mis en œuvre. Si de nouvelles techniques de laboratoire pouvaient permettre une mesure précise et rapide des caractéristiques variétales des semences, on pourrait imaginer une adaptation du système de certification qui, pour ces caractéristiques, s'affranchirait des contrôles en culture actuels pour basculer vers un contrôle de résultats sur les 
lots,avant mise en marché. Cependant, compte tenu des coûts inhérents à ces techniques, d'une part, et des enjeux financiers représentés par chaque lot de semences pour le secteur des oléagineux, d'autre part, le contexte actuel ne semble pas favorable à ce type d'évolution.

\section{Enjeux futurs de la certification}

Jusqu'à présent, les bénéficiaires de la certification étaient les agriculteurs, auxquels elle apporte des garanties variétales, technologiques et sanitaires des semences achetées, et les entreprises semencières, à qui la certification, comme base d'harmonisation, offre un moyen de libre échange dans l'Union européenne et, dans une certaine mesure, avec les pays tiers.

De nouveaux « clients » apparaissent désormais en aval : les industriels, qui intègrent de plus en plus I'utilisation de semences certifiées dans les cahiers des charges de leurs producteurs pour répondre aux attentes de leurs clients, notamment en termes de traçabilité, et les consommateurs, demandeurs de transparence, d'information sur l'origine des produits et de plus de qualité.

Nul doute que la certification saura s'adapter à ces nouvelles exigences pour permettre d'apporter de nouvelles garanties sur la teneur en composants spécifiques (acides gras, protéines, etc.), pour garantir le respect des seuils définis de présence fortuite d'OGM dans les semences de variétés conventionnelles, mais également pour garantir la pureté OGM de variétés utilisant cette technologie lorsque leur culture sera autorisée en Europe (encadré).

\section{Encadré}

OGM et certificationDans l'état actuel de la réglementation des semences, la certification ne garantit pas l'absence d'impuretés OGM dans les semences de variétés conventionnelles. Des discussions au niveau de l'Union européenne envisagent de mettre en place un seuil dit " de présence fortuite en OGM ", destiné à tolérer ces impuretés dans des proportions réduites, de la même manière qu'il existe des normes de pureté variétale pour les plantes morphologiquement différentes de la variété attendue.

Les négociations portent sur le niveau du seuil, que la filière semences française souhaiterait voisin de $1 \%$, mais que d'autres pays voudraient inférieur, voire égal à zéro. II semble cependant illusoire de vouloir s'affranchir complètement d'une présence d'impuretés OGM compte tenu des modes de fécondation de certaines espèces (pollen véhiculé par le vent ou les insectes et pouvant être déplacé sur des distances importantes).

Ce seuil attendu pourrait alors être intégré dans les garanties apportées par la certification mais nécessiterait de renforcer les règles de production en culture de certaines espèces et de mettre en place des analyses de contrôle.

Les semenciers réalisent déjà depuis plusieurs années des analyses destinées à s'assurer de l'absence d'OGM dans les semences mères utilisées qui, dans certains cas, peuvent provenir de pays où les cultures de variétés OGM sont autorisées. 
Cependant, si un seuil relativement bas peut s'envisager en France dans un environnement agricole quasiment indemne de cultures de variétés OGM, il serait beaucoup plus difficile à mettre en œuvre dans un contexte où les variétés OGM seraient cultivées à grande échelle. La possibilité de produire en France des semences de certaines espèces risquerait alors d'être remise en cause.

Illustrations

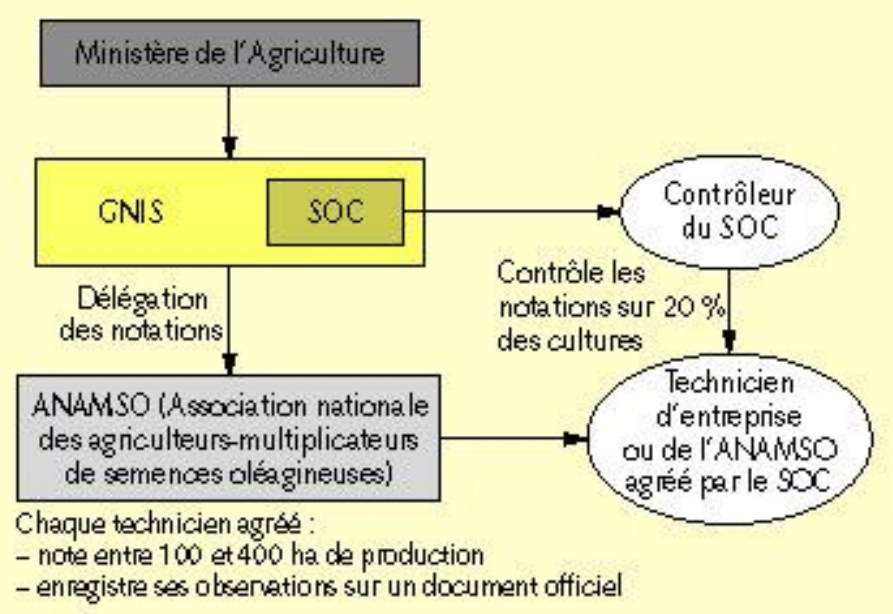

Figure. Oléagineux : organisation du contrôle des cultures de semences certifiées (SC).

\begin{tabular}{|c|c|c|c|}
\hline & $\begin{array}{c}\text { Colza oléagineux } \\
\text { d'hiver }\end{array}$ & Toumesol & Soja \\
\hline $\begin{array}{c}\text { Nombre d'entrepprises } \\
\text { productrices }\end{array}$ & 24 & 25 & 16 \\
\hline $\begin{array}{c}\text { Nombre de contrats } \\
\text { de productions }\end{array}$ & 560 & 1280 & 2940 \\
\hline $\begin{array}{c}\text { Surfaces présentées } \\
\text { au contrôle (ha) }\end{array}$ & 3190 & 5430 & 40 \\
\hline $\begin{array}{c}\text { Nombre de variétés } \\
\text { produites }\end{array}$ & $\begin{array}{c}126 \\
47 \text { géniteurs }\end{array}$ & $\begin{array}{c}172 \\
167 \text { géniteurs }\end{array}$ & 7380 \\
\hline $\begin{array}{c}\text { Quantités certifiées } \\
\text { (T) }\end{array}$ & 5760 & 7930 & \\
\hline
\end{tabular}

Certification des semences oléagineuses en France - 2000/2001 - (colza, tournesol, soja). 


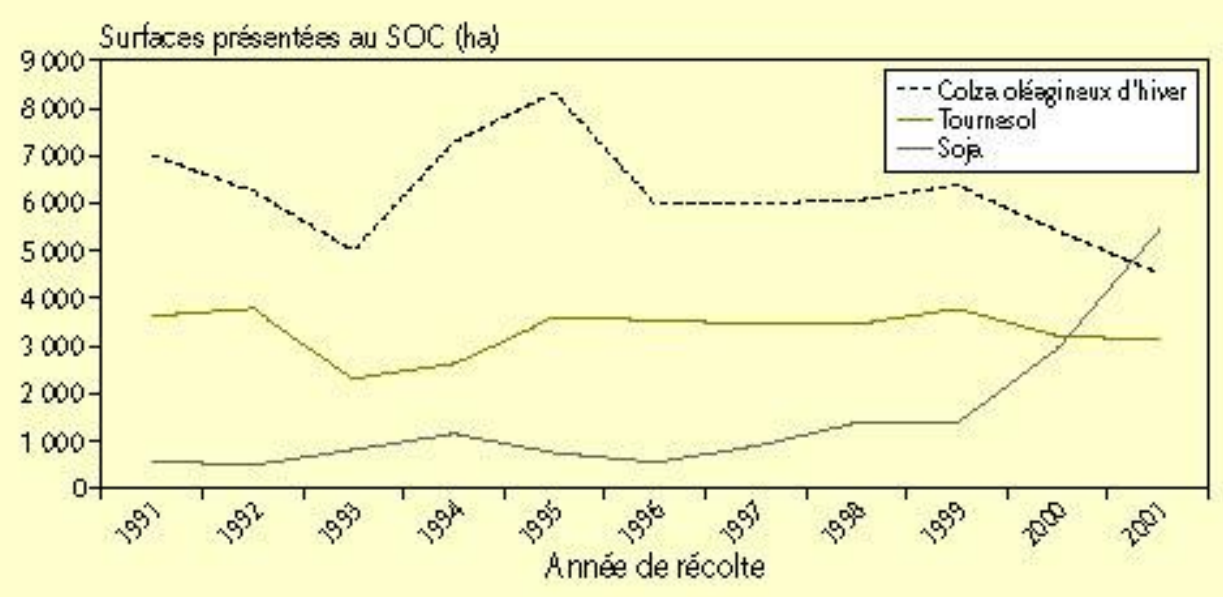

Semences oléagineuses : évolution des surfaces de production en France (colza, tournesol, soja). 